\title{
Diabetes and thalassaemia
}

\author{
Maria Barnard, ${ }^{1}$ Ploutarchos Tzoulis ${ }^{2}$ \\ ${ }^{1}$ The Whittington Hospital NHS Trust; ${ }^{2}$ Romilla Jones, Emma Prescott, Farrukh Shah, \\ The Whittington Hospital NHS Trust, Whittington Health, London, UK
}

\begin{abstract}
Diabetes is a significant complication of $\beta$-thalassaemia major. The aetiology includes iron overload causing $\beta$-cell destruction, autoimmunity, insulin resistance secondary to liver disease and development of type 1 or 2 diabetes. There are specific issues for patients with diabetes and thalassaemia which will be discussed here. Impaired carbohydrate metabolism must be detected early, to allow intensification of iron chelation. As life expectancy in thalassaemia rises, diabetic complications are seen. Optimising blood glucose and cardiovascular risk factor control is essential. Insulin remains critical for severely symptomatic patients. With milder hyperglycaemia, oral antidiabetic drugs are increasingly used. At Whittington Hospital, we wanted to address these issues. In 2005, we developed a unique Joint Diabetes Thalassaemia Clinic, where patients are reviewed jointly by specialist teams, including Consultant Diabetologist and Haematologist. The Joint Clinic aims to optimise diabetes, endocrine and thalassaemia care, while supporting patient self-management. A retrospective audit of the Joint Clinic (2005-09), showed improvement in glycaemic control, (Fructosamine falling from $344 \mathrm{umol} / /$ to $319 \mathrm{umol} / /$ ). We compared our cohort to the National Diabetes Audit for England (2007-08). Patients attending the Joint Clinic achieved better glycaemic control (target reached: 73\% Joint Clinic vs. 63\% Nationally), blood pressure control (target reached: 58\% Joint Clinic vs. 30\% Nationally) and cholesterol control (target reached: 81\% Joint Clinic vs. 78\% Nationally). $22.7 \%$ of our patients had $\geq 1$ microvascular complication. A significant proportion had endocrinopathies (86\% hypogonadism, 23\% hypoparathyroidism, 18\% hypothyroidism). Managing diabetes is one of the greatest challenges a person with thalassaemia can face. Training people to self-manage their diabetes and providing support from specialist teams working together are critical. The unique partnership working of our Joint Diabetes Thalassaemia Clinic allows these very complex patients to be managed effectively.
\end{abstract}

Correspondence: Maria Barnard

(C) Copyright M. Barnard and P. Tzoulis, 2013

Licensee PAGEPress, Italy

Thalassemia Reports 2013; 3(s1):e18

doi:10.4081/thal.2013.s1.e18

This article is distributed under the terms of the Creative Commons Attribution Noncommercial License (by-nc 3.0) which permits any noncommercial use, distribution, and reproduction in any medium, provided the original author(s) and source are credited.

Parts of this work were presented at the

"3rd Pan-European Conference on Haemoglobinopathies and Rare Anaemias", Limassol (Cyprus), 24-26 October 2012.

\section{Introduction}

Diabetes mellitus is a major global health challenge. In 2011, around 366 million people worldwide were living with diabetes.(1) That number is predicted to rise to 552 million by 2030 . Diabetes imposes a large economic burden on the individual and on healthcare. At least USD 465 billion dollars were spent on diabetes in 2011, accounting for $11 \%$ of total healthcare expenditure in the worldl. In the United Kingdom (UK), the current cost of direct patient care (including treatment, intervention and complications) for those living with diabetes is estimated at $£ 9.8$ billion.(2) There are further indirect costs associated with diabetes (such as those related to increased death and illness, work loss and the need for informal care) which are currently estimated at $£ 13.9$ billion in the UK2. The total cost associated with diabetes in the UK is estimated at $\$ 23.7$ billion and that is projected to increase to $\$ 39.8$ billion in $2035 / 62$.

Diabetes is a significant complication of $\beta$-thalassaemia major. The estimates of prevalence are variable, ranging between $1 \%$ to $21 \%$ of patients attending a thalassaemia service.(3,4) This variability partly relates to the introduction and application of effective iron chelation therapy and also relates to the ages of patients being studied, with lower rates in younger patients. Various aetiological factors may contribute to development of diabetes in thalassaemia. Transfusional iron overload is a key factor damaging pancreatic $\beta$-cells and causing diabetes. Risk factors for developing impaired glucose metabolism in thalassaemia include poor compliance with chelation therapy and advanced age at onset of chelation therapy.(5) Other possible aetiological factors include altered $\beta$-cell insulin secretion,(6) autoimmunity,(7) insulin resistance secondary to liver disease5 and Hepatitis $\mathrm{C}$ virus infection affecting glucose metabolism.(8) Furthermore, with the worldwide epidemic of diabetes, patients with thalassaemia could develop type 1 or type 2 diabetes, independently of their thalassaemia.

There are specific issues to consider in the management of patients with diabetes and thalassaemia. First, impaired carbohydrate metabolism and diabetes must be detected early and UK and international thalassaemia management guidelines advise regular screening with annual oral glucose tolerance tests from puberty or from age 10 years if there is a positive family history. $(9,10)$ This allows for prompt treatment of hyperglycaemia but also for intensification of iron chelation therapy, which may improve glycaemic control or prevent frank diabetes developing.(11)

A critical aim in diabetes care is the prevention, detection and management of diabetic complications, including macrovascular complications (cardiovascular disease, cerebrovascular disease, peripheral vascular disease) and microvascular complications (diabetic retinopathy, nephropathy and neuropathy). These complications can cause major patient morbidity and mortality and also account for around $80 \%$ of direct patient care costs in the UK2. As the life expectancy of patients with thalassaemia rises, these diabetic complications are being seen, particularly microvascular disease.(12,13) Optimising blood glucose and cardiovascular risk factor control is essential.

The medical treatment of diabetes in thalassaemia is dependent on 
how early diabetes is diagnosed and how severe the $\beta$-cell damage and subsequent insulin deficiency are. Insulin remains the main treatment for glycaemic control in the severely hyperglycaemic patient.(9) For patients with impaired glucose tolerance or diabetes detected early with mild hyperglycaemia, a focus on self-management and lifestyle changes is important, particularly following a healthy diet and weight reduction, where applicable. In these patients, oral antidiabetic drugs are also increasingly prescribed. The use of metformin,(14) sulphonylureas (15) or acarbose (16) in diabetes and thalassaemia has been described, but there is very limited published data on the role of these different agents.

The impact of diabetes in thalassaemia must be considered in many other situations. In pregnancy, diabetes in the mother can lead to a four-fold increased risk of foetal anomaly and three-fold increase in perinatal mortality.(10) In patients with cardiac complications, diabetes can adversely affect cardiac function and should be monitored.(10) Glycaemic control should be improved but care should be taken with insulin initiation or intensification to avoid fluid retention and heart failure.(17) The physiological stress associated with surgery can lead to significant deterioration in glycaemic control, which itself can lead to increased morbidity and mortality from surgery. Diabetes must be excluded or adequately managed around the time of surgery in patients with thalassaemia.(10)

Living with diabetes and thalassaemia can also have a major psychological impact, with feelings of treatment burden, impact on daily life, feeling of difference, dependence, feeling of damage and anxiety.(11) Healthcare professionals need to be able to draw out and discuss these issues with patients. They also need to support patients to manage their diabetes and to enable patients to apply these self-management skills to lead a full and rich life.

We wanted to address these specific issues for patients living with both diabetes and thalassaemia. We developed a unique Joint Diabetes Thalassaemia Clinic, which we will now describe.

\section{Methodology}

In 2005, at Whittington Hospital, we set up and developed a Joint Diabetes Thalassaemia Clinic. Prior to this, patients with diabetes and thalassaemia could be seen by different diabetes clinicians, both senior and more junior, in a variety of diabetes clinics. For patients with complex medical needs, this was providing an inadequate level of care.

The aims of the Joint Diabetes Thalassaemia Clinic are: to provide high quality diabetes, endocrine and haematology care; to optimise metabolic control; to support patient self-management; and to support partnership working, both between the specialist teams and between patients and clinicians.

At the Joint Diabetes Thalassaemia Clinic, patients are seen jointly by Consultant Diabetologist (Dr Maria Barnard), Consultant Haematologist (Dr Farrukh Shah), Diabetes Specialist Nurse (Romilla Jones) and Haematology Specialist Nurse (Emma Prescott). A specified Diabetes Dietitian supports the clinic. There is access to Whittington structured education courses principally provided for patients with type 1 diabetes, including training for carbohydrate counting and insulin dose adjusting. Patients can be referred to a specified Clinical Psychologist, with experience in enabling patients to cope with issues relating to thalassaemia.

Appointments at the Joint Diabetes Thalassaemia Clinic with the Consultants are available every 3 months, but generally patients are reviewed in the Joint Clinic once every 6-9 months. Interim reviews to optimise glycaemic control and patient knowledge and self-management skills are provided by the Diabetes Specialist Nurse and Diabetes Dietitian.

The UK and international guidelines for the clinical care of adults with thalassaemia state that patients with diabetes should be managed according to standard recommendations for diabetes. $(10,11)$ In the Joint Clinic, we therefore aim to complete a full diabetes annual review once a year, addressing the 9 Key care Processes for diabetes, derived from national guidance. $(18,19)$ These are measurement of glycaemic control, blood pressure, serum cholesterol, serum creatinine, urinary albumin and weight, foot assessment, smoking status assessment and retinal screening. We adopted management targets for control of blood glucose, blood pressure and lipids consistent with national guidance.(18,19) We assess glycaemic control using both patient home blood glucose monitoring results and Fructosamine levels,(11) since HbAlc levels can be inaccurate in patients receiving repeated transfusions and with haemoglobinopathies. Our biochemistry laboratory provides a correction factor for converting Fructosamine to an equivalent HbAlc level (where Fructosamine $322 \mathrm{umol} / /$ is equivalent to $\mathrm{HbAlc} 7 \%$ ).

Service performance was assessed by retrospective analysis of data from 81 consultations of 22 patients attending the Joint Diabetes Thalassaemia Clinic between December 2005 to December 2009. The results of our cohort were compared to the results of the National Diabetes Audit for England in 2007-2008.(20) The objectives of the audit were to compare the proportion of patients in the Joint Diabetes Thalassaemia Clinic who received the key processes of diabetes care, who achieved treatment targets and who had microvascular and macrovascular complications. The prevalence of other endocrinopathies was also measured.

\section{Results}

From December 2005-2009, 22 patients were reviewed in the Joint Diabetes Thalassaemia Clinic. They had 81 consultations over the four year period. The median number of consultations was 3.5 per patient with a significant spread (from 1 consultation in 5 patients to 9 consultations in 1 patient). Where patients had only 1 or 2 reviews in the Joint Clinic, follow-up data was not always available to assess changes in parameters.

The patient median age was 39 years (range 28 - 59 years old) (Table 1). Thirteen were female (59\%) and 9 were male (41\%). Patient ethnic origin was 8 Greek or Greek Cypriot people and 14 South Asian people (Indian, Pakistani, Banglasdeshi). The median duration of diabetes was 13 years

Table 1. Description of patients attending the joint clinic.

\begin{tabular}{|c|c|c|c|c|c|c|}
\hline Age & \multicolumn{2}{|l|}{ Ethnicity } & Diabetes duration & Age onset diabetes & \multicolumn{2}{|c|}{ Diabetes therapy } \\
\hline 39 years* $(28-59 y)$ & Greek/Greek Cypriot & $36 \%$ & 13 years* & 21 years* & Insulin & $73 \%$ \\
\hline & South Asian & $64 \%$ & $(<1-29 y)$ & $(10-40 y)$ & Oral agents & $14 \%$ \\
\hline & & & & Diet only & $14 \%$ & \\
\hline
\end{tabular}

*median (range) 
(range $<1$ year - 29 years). The median age of onset of diabetes was 21 years of age (range 10 - 40 years), with 9 patients (41\%) diagnosed before the age of 19 years.

At the first appointment, 4 patients were smokers (18\%). The median body mass index (BMI) was $24.8 \mathrm{~kg} / \mathrm{m} 2$ and 11 patients (50\%) were overweight (BMI $25-30 \mathrm{~kg} / \mathrm{m} 2$ ) or obese (BMI $>30 \mathrm{~kg} / \mathrm{m} 2$ ). The median ferritin at first appointment was $1827 \mathrm{ug} / \mathrm{l}$ (range 600 - $6143 \mathrm{ug} / \mathrm{dl})$. For patients with Joint Clinic follow-up data available, 33\% achieved a reduction in ferritin of $>10 \%$.

Patients were on medications relating to diabetes and management of complications. 16 patients (73\%) were on insulin. 6 patients $(27 \%)$ were on oral antidiabetic drugs (metformin, glimepiride). 3 patients (14\%) were on diet control alone. 5 patients (23\%) were on antihypertensive medication (angiotensin-converting enzyme [ACE] inhibitors or angiotensin-II receptor antagonists). 5 patients (23\%) were on lipid lowering agents (statins or fibrates). 7 patients (32\%) were on antiplatelet or anticoagulant therapy (aspirin or warfarin).

In patients attending the Joint Clinic, the Key Processes for diabetes care were performed at a rate much greater than that achieved in the National Diabetes Audit for England (2007-2008)20 (Table 2).This included better rates of measurement of Fructosamine, serum cholesterol, serum creatinine, urinary albumin-to-creatinine ration (ACR) and weight (BMI), foot assessment and smoking status assessment.

In patients attending the Joint Clinic, there was a greater achievement of management targets compared to that achieved in the National Diabetes Audit for England (2007-2008)20 (Table 3). In our cohort, 73\% achieved Fructosamine $<345 \mathrm{umol} / \mathrm{l}$ (equivalent to $\mathrm{HbAlc}<7.5 \%$ ) and $55 \%$ achieved Fructosamine $<322 \mathrm{umol} / /$ (equivalent to HbAlc $<7.0 \%$ ). In the National Diabetes Audit, $63 \%$ of patients achieved HbAlc $<7.5 \%$. In our cohort, $58 \%$ achieved blood pressure $<135 / 75 \mathrm{mmHg}$. In the National Diabetes Audit, 30\% of patients achieved blood pressure $<135 / 75 \mathrm{mmHg}$. In our cohort, $81 \%$ achieved total cholesterol $<5.0$

Table 2. Performance of Key Care Processes at all Joint Clinic reviews compared to National Diabetes Audit for England.

\begin{tabular}{lcc} 
Care Process & $\begin{array}{c}\text { Performance of Key Care Processes } \\
\text { Joint Clinic } \\
(2005-2009)\end{array}$ & $\begin{array}{c}\text { National Diabetes Audit } \\
(2007-2008)\end{array}$ \\
Fructosamine/HbAlc & $97.5 \%$ & $91.1 \%$ \\
Serum cholesterol & $91.1 \%$ & $89.9 \%$ \\
\hline Serum creatinine & $100 \%$ & $91.2 \%$ \\
Urinary albuminuria & $91.1 \%$ & $62.7 \%$ \\
\hline Weight/Body mass index & $97.5 \%$ & $88.8 \%$ \\
Blood pressure (BP) & $80.4 \%$ & $93.7 \%$ \\
\hline Foot assessment & $89.2 \%$ & $77.1 \%$ \\
Smoking status & $89.2 \%$ & $86.5 \%$ \\
\hline
\end{tabular}

Table 3. Rate of achievement of treatment targets in Joint Clinic compared to National Diabetes Audit for England (2007-2008).

Target Percentage of patients achieving
treatment target
Joint Clinic National Diabetes Audit

Fructosamine $<345 \mathrm{umol} / \mathrm{l}$

\begin{tabular}{lll} 
HbAlc $<7.5 \%$ & $72.7 \%$ & $62.9 \%$ \\
$\mathrm{BP}<135 / 75 \mathrm{mmHg}$ & $57.9 \%$ & $30.1 \%$ \\
\hline Total cholesterol $<5.0 \mathrm{mmol} / \mathrm{l}$ & $82.1 \%$ & $78.0 \%$ \\
\hline
\end{tabular}

$\mathrm{mmol} / /$ and $69 \%$ achieved total cholesterol $<4.0 \mathrm{mmol} /$. In the National Diabetes Audit, $78 \%$ of patients achieved total cholesterol $<5.0 \mathrm{mmol} /$.

Where follow-up data was available, improvements were achieved in metabolic control (Table 4). Fructosamine levels fell by $25 \mathrm{umol} / /$ in the first year (median Fructosamine at first appointment $344 \mathrm{umol} / \mathrm{l}$ vs. median Fructosamine at 1 year follow-up 319 umol/l). $44 \%$ of patients achieved a reduction in Fructosamine of $>30 \mathrm{umol} / \mathrm{l}$ (equivalent to a reduction in $\mathrm{HbA1c}$ of $0.7 \%$ ). Median blood pressure readings did not reduce. $31 \%$ of patients had a reduction in systolic blood pressure by $>10 \mathrm{mmHg}$ and $25 \%$ of patients had an increase in diastolic blood pressure by $>10 \mathrm{mmHg}$. Total cholesterol levels fell by -0.3 $\mathrm{mmol} / \mathrm{l}$ in the first year (median total cholesterol at first appointment $3.8 \mathrm{mmol} / \mathrm{l} v \mathrm{~s}$. median total cholesterol at 1 year follow-up $3.5 \mathrm{mmol} / \mathrm{l}$ ). $23 \%$ of patients achieved a reduction in total cholesterol of $>0.4$ $\mathrm{mmol} /$.

Microvascular complications were present in this cohort. 3 patients (13.6\%) had microalbuminuria. 3 patients (13.6\%) had diabetic retinopathy. Overall 5 patients $(22.7 \%)$ had at least one microvascular complication. 1 patient had Charcot Neuroarthropathy and foot ulcer. 2 patients $(9.1 \%)$ had cataracts.

No macrovascular complications (cardio-, cerebro- or peripheral-vascular disease) were documented. All patients had all peripheral pulses palpable in their feet and no amputations had occurred. No episodes of diabetic hyperglycaemic emergencies were documented (no diabetic ketoacidosis or hyperglycaemic hyperosmolar state).

A significant majority of the patients had other endocrinopathies (Table 5). Hypogonadism was present in 19 patients (86\%), who were all on hormone replacement therapy, including testosterone. Hypothyroidism was present in 4 patients (18\%), who were all on thyroxine. Hypoparathyroidsim was present in 5 patients (23\%). 12 patients (55\%) were taking calcitriol. No glucocorticoid or growth hormone deficiency was documented.

Table 4. Change in metabolic control in Joint Clinic (follow-up data available).

\begin{tabular}{lccc} 
Parameter & First appointment & 1 year follow-up & Change \\
Fructosamine & $344 \mathrm{umol} / \mathrm{l}$ & $319 \mathrm{umol} / \mathrm{l}$ & $-25 \mathrm{umol} / \mathrm{l}$ \\
$\mathrm{BP}$ & $122 / 70 \mathrm{mmHg}$ & $124 / 77 \mathrm{mmHg}$ & $+2 / 7 \mathrm{mmHg}$ \\
\hline Total cholesterol & $3.8 \mathrm{mmol} / \mathrm{l}$ & $3.5 \mathrm{mmol} / \mathrm{l}$ & $-0.3 \mathrm{mmol} / \mathrm{l}$ \\
\hline
\end{tabular}

Table 5. Percentage of patients attending Joint Clinic with other endocrinopathies.

\begin{tabular}{lc}
\hline Endocrinopathy & Prevalence in patients in Joint Clinic \\
Hypogonadism & $86 \%$ \\
- Hypogonadotrophic hypogonadism & $59 \%$ \\
- Primary hypogonadism & $27 \%$ \\
Hypothyroidism & $18 \%$ \\
\hline Hypoparathyroidism & $23 \%$ \\
Osteopenia & $14 \%$ \\
\hline Osteoporosis & $55 \%$ \\
Glucocorticoid deficiency & - \\
\hline Growth hormone deficiency & -
\end{tabular}




\section{Discussion}

Our audit review has confirmed that our Joint Diabetes Thalassaemia Clinic is very effective at providing high quality diabetes care to the most complex patients. The standard of care provided was significantly higher than that found in the National Diabetes Audit. There was greater performance of the key care processes in diabetes management. Further, a greater proportion of our Joint Clinic patients achieved the targets for diabetes management, including glycaemic control, blood pressure control and total cholesterol levels compared to the national data.

The age of onset of diabetes in our patients attending the Joint Clinic was young (median age 21 years) and a significant proportion were diagnosed before the age of 19 years (41\%). This suggests that tissue iron overload and poor iron chelation can significantly damage $\beta$-cell function at an early age. It is essential that effective iron chelation therapy is instigated early. Patient self-care issues, particularly adherence to demanding chelation therapy regimens, must be discussed, addressed and managed.

Improvements in metabolic control were seen, with $25 \mathrm{umol} / /$ reduction in Fructosamine levels after one year of follow-up (equivalent to a reduction in $\mathrm{HbAlc}$ of $0.6 \%$ ). A reduction in tissue iron overload was also suggested by a reduction of serum ferritin levels in $33 \%$ of the clinic population. This suggests an overall improvement in care.

The rate of diabetes complications in our Joint Clinic patients was low, with no documented macrovascular disease and 1-2 microvascular complications present in $22.7 \%$. This cohort is fairly young (median age 39 years). As the life expectancy of patients with thalassaemia rises, this will also expose our patients potentially to many more years of hyperglycaemia and diabetes. Patients in the Joint Clinic were being actively medically managed. This was evidenced by the significant use of glucose-lowering therapy (86\%), antihypertensive medication (23\%) and lipid lowering agents (23\%). Sustaining improvements in metabolic control and controlling cardiovascular risk factors will be critical in the future for preventing diabetic complications.

These patients have complex medical needs. In addition to their thalassaemia and diabetes, there was a high proportion with other endocrinopathies. This suggests that in these patients, poor chelation therapy in the past, including poor compliance with treatment regimens, caused significant tissue iron overload, with widespread damage and health consequences. Some of these patients were also contending with significant psychological issues, including anxiety and depression. Healthcare professionals in the Joint Clinic were able to support them or offer them further counselling with our dedicated Clinical Psychologist.

There are a variety of factors in the Joint Clinic that may be contributing to high quality care and improved metabolic control. The doctor's input to the clinic is provided by senior medical staff (Consultant Diabetologist and Consultant Haematologist), with experience and knowledge regarding diabetes, thalassaemia and new treatment options. All the members of the specialist teams providing the Joint Clinic have been involved from an early stage. This has provided continuity of care and patients know and are familiar with the healthcare professionals looking after them.

Through the diabetes professionals involved, patients have access to diabetes education, including training on carbohydrate counting and insulin dose adjustment. This education is provided on a one-to-one basis. However, patients from the Joint Clinic who are on multiple daily insulin injection therapy have also been invited to attend the group structured education course provided for patients with type 1 diabetes at the Whittington Hospital. They have greatly enjoyed and highly valued this skills training. Further, the Joint Clinic provides significant educational opportunities for the healthcare professionals involved. This means that they use and spread this knowledge in other situations, particularly when the diabetes or haematology team are seeing patients with thalassaemia or diabetes respectively, outside the Joint Clinic.

\section{Conclusions}

The unique partnership working of our Joint Diabetes Thalassaemia Clinic allows patients with complex medical problems to be effectively managed. Patients have easy access to senior specialist clinicians supported by a multidisciplinary team working together. In addition to high level medical information and advice, patients are supported to selfmanage their diabetes and thalassaemia, to sustain improvements in metabolic control and health. Managing diabetes is one of the greatest challenges a person with thalassaemia can face. Our Joint Diabetes Thalassaemia Clinic enables these very complex patients to effectively manage their physical and psychological long-term health.

\section{References}

1. International Diabetes Federation. IDF Diabetes Atlas. Fifth Edition 2011. Available at: http://www.idf.org/diabetesatlas

2. Hex N, Bartlett C, Wright D, Taylor M, Varley D. Estimating the current and future costs of Type 1 and Type 2 diabetes in the UK, including direct health costs and indirect societal and productivity costs. Diabet Med. 2012 Jul;29(7):855-62

3. Borgna-Pignatti C, Rugolotto S, De Stefano P, Zhao H, Cappellini MD, Del Vecchio GC, Romeo MA, Forni GL, Gamberini MR, Ghilardi R, Piga A, Cnaan A. Survival and complications in patients with thalassemia major treated with transfusion and deferoxamine. Haematologica 2004;89(10):1187-93

4. Cunningham MJ, Macklin EA, Neufeld EJ, Cohen AR. Thalassemia Clinical Research Network. Complications of beta-thalassemia major in North America. Blood 2004;104(1):34-9

5. Gamberini MR, Fortini M, De Sanctis V, Gilli G, Testa MR. Diabetes mellitus and impaired glucose tolerance in thalassaemia major: incidence, prevalence, risk factors and survival in patients followed in the Ferrara Center. Pediatr Endocrinol Rev. 2004;2 Suppl 2:285-91

6. Angelopoulos NG, Zervas A, Livadas S, Adamopoulos I, Giannopoulos D, Goula A, Tolis G. Reduced insulin secretion in normoglycaemic patients with beta-thalassaemia major. Diabet Med. 2006;23(12):1327-31

7. Monge L, Pinach S, Caramellino L, Bertero MT, Dall'omo A, Carta Q. The possible role of autoimmunity in the pathogenesis of diabetes in B-thalassemia major. Diabetes MeTable 2001;27(2 Pt 1):149-54

8. Mowla A, Karimi M, Afrasiabi A, De Sanctis V. Prevalence of diabetes mellitus and impaired glucose tolerance in beta-thalassemia patients with and without hepatitis $\mathrm{C}$ virus infection. Pediatr Endocrinol Rev. 2004;2 Suppl 2:282-4

9. Thalassaemia International Federation. Guidelines for the Clinical Management of Thalassaemia. 2nd Revised Edition 2008. Available at: http://www.thalassaemia.org.cy/publications.html

10. United Kingdom Thalassaemia Society. Standards for the Clinical Care of Children and Adults with Thalassaemia in the UK. 2nd Edition 2008. Available at: http://www.ukts.org/pdf.html

11. Berdoukas V, Farmaki K, Wood JC, Coates T. Iron chelation in thalassemia: time to reconsider our comfort zones. Expert Rev Hematol. 2011;4(1):17-26 
12. Incorvaia C, Parmeggiani F, Mingrone G, Sebastiani A, De Sanctis V. Prevalence of retinopathy in diabetic thalassaemic patients. J Pediatr Endocrinol MeTable 1998;11 Suppl 3:879-83

13. Loebstein R, Lehotay DC, Luo X, Bartfay W, Tyler B, Sher GD. Diabetic nephropathy in hypertransfused patients with beta-thalassemia. The role of oxidative stress. Diabetes Care 1998;21(8):1306-9

14. Dhouib N, Turki Z, Mellouli F, Ouederni M, Yahiaoui S, Nagi S, Ben Slama C, Bejaoui M. Efficacy of metformin in the treatment of diabetes mellitus complicating thalassemia major. Tunis Med. 2010;88(2):136.

15. Ladis V, Theodorides C, Palamidou F, Frissiras S, Berdousi H, Kattamis C. Glucose disturbances and regulation with glibenclamide in thalassemia. J Pediatr Endocrinol MeTable 1998;11 Suppl 3:871-8

16. Mangiagli A, Campisi S, De Sanctis V, Nicoletti MC, Cardinale G, Galati MC, Raiola G, Rigano P, Saviano A; Study Group of the Italian Pediatric and Diabetes Soceity (SIEDP) on Endocrine Complications in Non-Endocrine Disease. Effects of acarbose in patients with beta-thalassaemia major and abnormal glucose homeostasis. Pediatr Endocrinol Rev. 2004;2 Suppl 2:276-8

17. Konrad D, Daneman D, Kirby M, Wherrett D. Cardiac failure after initiation of insulin treatment in diabetic patients with beta-thalassemia major. J Pediatr. 2003;143(4):541-2

18. National Institute for Clinical Excellence. Type 1 diabetes: diagnosis and management of type 1 diabetes in children, young people and adults. Clinical Guideline 15, July 2004. Available at: http://guidance.nice.org.uk/CG15/NICEGuidance/pdf/English

19. The National Collaborating Centre for Chronic Conditions. Type 2 diabetes. National clinical guideline for management in primary and secondary care (update). Published by Royal College of Physicians of London 2008. Available at: http://guidance.nice.org. uk/CG66/Guidance/pdf/English

20. NHS Information Centre. National Diabetes Audit. Key findings about the quality of care for people with diabetes in England and Wales. Report for the audit period 2007-2008. Leeds: NHS IC, 2008. Available at: http://www.ic.nhs.uk/nda

\section{Oral presentation}

Oral presentation is available online 